\title{
Acreditación Nacional y Regional de las carreras de Ingeniería. EI MARCA como herramienta clave para la integración ${ }^{1}$
}

\author{
National and regional accreditation of engineering degree courses \\ The MARCA as a key tool for integration
}

\section{Silvana Lorena Lagoria ${ }^{2}$ silvanal_Ic@yahoo.com.ar Consejo Nacional de Investigaciones Científicas y Técnicas (CONICET) Universidad Nacional de Córdoba (UNC) Universidad Blas Pascal (UBP) Argentina}

\author{
Volumen 9, Número 1 \\ 30 de mayo de 2018 \\ pp. 55 - 82
}

Recibido: 6 de mayo de 2017

Aprobado: 7 de febrero de 2018

\begin{abstract}
${ }^{1}$ Este artículo presenta parte de los resultados obtenidos en una investigación de mayor alcance titulada "Acreditación de carreras de grado en el ámbito nacional y regional. El caso de las ingenierías" (Lagoria, 2016). En dicha investigación se analizaron desde una perspectiva comparada los cambios que se producen en las carreras de grado al transitar los procesos de control de calidad en Argentina y el MERCOSUR. También se incluyó un anexo sobre la experiencia de las carreras de Ingeniería Electrónica en la Unión Europea (UE), esto resultó un aporte enriquecedor para conocer una experiencia de acreditación regional en un contexto diferente al latinoamericano.

2 Silvana Lorena Lagoria es Dra. en Estudios Sociales de América Latina, Magister. en Gestión para la Integración Regional, Especialidad en Políticas Públicas y Evaluación de la Educación Superior, Prof. y Lic. en Ciencias de la Educación y Maestra en Música. En la actualidad es Becaria Postdoctoral CONICET y docente en la Universidad Nacional de Córdoba (UNC) y Universidad Blas Pascal (UBP). E-mail: silvanal Ic@yahoo.com.ar
\end{abstract}

\footnotetext{
Acreditación Nacional y Regional de las carreras de Ingeniería. El MARCA como herramienta clave para la integración

Silvana Lorena Lagoria

DOI : http://dx.doi.org/10.22458/caes.v9i1.1771
} 


\title{
Resumen
}

Este artículo comienza con una introducción donde se describe la estrategia metodológica, se delinea el marco conceptual que orientó el análisis del caso propuesto en la investigación de origen y se fundamenta la importancia de la investigación realizada. A continuación, se desarrollan tres apartados donde se reconstruye brevemente el contexto en que se comprenden los procesos de acreditación de carreras de grado, se comentan de manera crítica los principales hallazgos de la investigación en relación con los procesos de acreditación en el nivel nacional (Argentina) y regional (MERCOSUR y Unión Europea). Para finalizar, se presentan algunas reflexiones sobre la experiencia de las carreras analizadas y se rescata el valor del MARCA para afianzar la integración educativa en la región.

Palabras clave: Acreditación; ingeniería; MERCOSUR; Unión Europea (UE); MARCA.

\begin{abstract}
This article begins with an introduction describing the methodological strategy, outlining the conceptual framework that guided the analysis of the case proposed in the original investigation and based the importance of the research carried out. Then, three sections are developed that briefly reconstruct the context in which the processes of accreditation of degree courses are understood, the main findings of the research in relation to accreditation processes at the national level (Argentina) And regional level (MERCOSUR and European Union) are critically discussed. Finally, some reflections on the experience of the degree courses analyzed are presented and the value of the MARCA is reinforced in order to consolidate the educational integration in the region.
\end{abstract}

Keywords: Accreditation; engineering; MERCOSUR; European Union (EU); MARCA.

Acreditación Nacional y Regional de las carreras de Ingeniería. El MARCA como herramienta clave para la integración

Silvana Lorena Lagoria

DOI : http://dx.doi.org/10.22458/caes.v9i1.1771

\section{(c) (i) (8)}




\section{Introducción}

Desde una perspectiva política, los procesos de acreditación de carreras de grado son el medio por el cual el Estado se propone controlar, garantizar y dar fe pública de la calidad de la oferta en educación superior. Además, desde una perspectiva sociológica, se considera que estos procesos contribuyen a afianzar una cultura de la calidad.

Como sabemos, las políticas definidas en el contexto macro se van impregnando, durante su puesta en marcha, de intereses y particularidades propias del accionar de los actores. Por tal motivo, este artículo se propone indagar acerca del modo en que los procesos de acreditación nacional y regional repercuten en aspectos organizativos y culturales de las carreras de Ingeniería y poder desentrañar desde adentro los "efectos" (Ball, 2002) ${ }^{3}$ de las macropolíticas que circunscriben a las instituciones de educación superior.

Este propósito conlleva a indagar más allá de los documentos donde se registra el proceso de acreditación y avanzar sobre las opiniones y perspectivas de los actores institucionales que vivenciaron el proceso. En tal sentido, la estrategia metodológica se estructuró en torno a un estudio predominantemente cualitativo que se complementó con algunos datos cuantitativos necesarios para contextualizar la especificidad de la realidad analizada.

La recolección de información se realizó a través de entrevistas semiestructuradas a informantes claves, principalmente, dirigidas a los secretarios académicos $\mathrm{y} / \mathrm{o}$ directores de carrera y a docentes. Esta elección se fundamentó en que dichos actores se desempeñan en el ámbito institucional específico donde se gestionan y ponen en marcha los procesos de acreditación.

${ }^{3}$ Ball (2002) señala que las políticas poseen efectos y no sólo resultados e identifica "efectos de primer orden", más relacionados con cambios en las prácticas.

Acreditación Nacional y Regional de las carreras de Ingeniería. EI MARCA como herramienta clave para la integración

Silvana Lorena Lagoria

DOI : http://dx.doi.org/10.22458/caes.v9i1.1771 
También se incluyó un análisis bibliográfico e investigación documental que permitieron especificar y conocer los procesos más amplios en que se ven implicadas las carreras cuando participan de los mecanismos de aseguramiento de la calidad. Principalmente, se consideraron documentos referidos a las carreras y a su participación en el proceso nacional y regional de acreditación.

Para delimitar el campo que abarcará el estudio, y por tratarse de una particularidad que incorporan las vivencias y las acciones de los participantes, se optó por emplear el estudio de caso (Stenhouse, 1990).

Asimismo, se recurrió al método comparativo con el objeto de comprender mejor la particularidad de cada realidad (tanto a nivel nacional como regional) y establecer relaciones entre ellos, es decir, observar conexiones en condiciones variables que es lo propio de la comparación como método en las ciencias sociales.

Teóricamente, nos posicionamos desde la concepción del cambio en educación superior tal como lo concibe Krotsch (2003), quien integra las posturas de Durkheim, Bourdieu y Clark para construir un modelo analítico del cambio institucional.

Para comprender los efectos de las políticas en su puesta en marcha nos resultó de utilidad recurrir al nuevo referencial teórico que propone Stephen Ball, denominado "perspectiva de los ciclos de la política (policy cycle approach) (...), 'caja de herramientas' para el análisis de la trayectoria de la política educativa" (Miranda, 2011: 105).

Acreditación Nacional y Regional de las carreras de Ingeniería. El MARCA como herramienta clave para la integración

Silvana Lorena Lagoria

DOI : http://dx.doi.org/10.22458/caes.v9i1.1771 
Tal como afirma Miranda (2011), en el ciclo de la política, Ball identifica cinco contextos donde las políticas son producidas, reproducidas, creadas y ejecutadas de manera interrelacionada, no lineal ni secuencialmente. Estos contextos son: "contexto de influencia, contexto de la producción del texto político, contexto(s) de la práctica, contexto de los resultados y contextos de la estrategia política" (Miranda, 2011: 109).

Si bien para el análisis político es importante tener en cuenta los cinco contextos que propone el autor, en este artículo nos resultó de mayor importancia comprender el "contexto de la práctica" porque allí es donde podemos identificar las acciones por las cuales el texto político es puesto en marcha y "está sujeto a interpretaciones, es recreado, reinterpretado, sufre un proceso de traducción por parte de los actores centrales de la práctica: los practitioners/docentes" (Miranda, 2011:115). Ball reconoce aquí que la cultura e historia institucionales, y las prácticas acumuladas son los elementos que determinan aquellas reinterpretaciones y significaciones que le atribuyen los actores (lectores) a las políticas al ponerlas en acto.

Al respecto, en una entrevista concedida a Mainardes y Marcondes (2009), Ball afirma que las políticas no son implementadas, ya que considerarlas de tal modo implicaría verlas de manera lineal y sistemática.

Al pretender desentrañar significados del nivel intrínseco de las instituciones, nos introducimos en el campo de la sociología de las organizaciones y de la cultura. En esa área, citamos autores como Crozier y Friedberg (1990), Geertz (1992) y Thompson (1990) para comprender conceptos que resultaron relevantes, tales como: campo, cambio, cultura, y la forma en que ellos se relacionan.

Acreditación Nacional y Regional de las carreras de Ingeniería. El MARCA como herramienta clave para la integración

Silvana Lorena Lagoria

DOI : http://dx.doi.org/10.22458/caes.v9i1.1771 
El foco del análisis estuvo puesto en lo que sucede en el nivel operativo de las prácticas y las modificaciones que se registran en respuesta al proceso de acreditación nacional y regional. Las carreras que formaron parte de este estudio son cuatro: Ingeniería Electrónica de la Facultad Regional Córdoba de la Universidad Tecnológica Nacional (FRC-UTN), Ingeniería Industrial de la FRC-UTN, Ingeniería Electrónica de la Universidad Nacional de Córdoba (UNC) e Ingeniería Industrial de la UNC.

El objetivo de este artículo es presentar desde una perspectiva crítica los resultados del análisis documental y de la información de campo en torno a las categorías de "cambio", entendido como mejoras a partir del proceso de acreditación, y de "cultura de la calidad". Ambos conceptos fueron analizados desde el material empírico alusivo a las prácticas desarrolladas por los actores para dar respuesta a las demandas del proceso de acreditación a la luz del marco teórico definido.

La importancia de la temática abordada se funda en varias razones. En primer lugar, en que en el contexto de cambio global emergen nuevas inquietudes y nuevas realidades de la educación superior latinoamericana que continúan siendo poco exploradas y demandan ser analizadas. Tal es el caso del tema del control de la calidad de la educación superior latinoamericana.

En segundo lugar, la acreditación de carreras de grado es una temática crucial pues atraviesa las fronteras nacionales para constituirse en asuntos regionales, a la vez que se constituye en un instrumento que redibuja desde una perspectiva diferente las fronteras que la globalización va borrando. Con ello nos referimos a que el proceso de acreditación

Acreditación Nacional y Regional de las carreras de Ingeniería. El MARCA como herramienta clave para la integración

Silvana Lorena Lagoria

DOI : http://dx.doi.org/10.22458/caes.v9i1.1771 
trasciende el nivel nacional y establece nuevos márgenes junto con los procesos de integración regional, contribuyendo al control de la calidad de la educación superior en los bloques regionales que se van constituyendo. Por ello, la acreditación de carreras de grado se torna un tema ideal para un análisis de alcance macro, meso y micro, gestado en el seno del contexto global.

$\mathrm{Y}$, en tercer lugar, porque el proceso de acreditación de carreras de grado debe comprenderse y analizarse como realidad particular emergente de un contexto de cambios globales que involucra no sólo a nuestro país, sino al extenso territorio latinoamericano y mundial. Por tal motivo, se torna un tema de interés para ser abordado desde la metodología comparada, ya que ésta, hoy en día, busca expandir su terreno atendiendo al nuevo escenario regional e internacional, algo que no ocurría en sus comienzos, pues se dedicaba al estudio de los sistemas educativos nacionales (Green, 2003; Yang, 2010). Desde este punto de vista, estamos contribuyendo a la construcción de conocimiento en educación superior desde una perspectiva comparada.

Con la intención de aportar a un debate en torno a la acreditación regional local, se incluye en el análisis una mirada hacia la experiencia de acreditación en el bloque Unión Europea.

\section{La acreditación en Argentina y el MERCOSUR}

Para comprender el análisis que se desarrolla a continuación, es preciso realizar algunas breves especificaciones en torno al contexto.

Con la globalización, a fines de 1980 y principios de 1990, fueron ganando terreno en las políticas educativas latinoamericanas las nociones de productividad, competitividad y calidad

\footnotetext{
Acreditación Nacional y Regional de las carreras de Ingeniería. EI MARCA como herramienta clave para la integración

Silvana Lorena Lagoria

DOI : http://dx.doi.org/10.22458/caes.v9i1.1771
} 
como condición de acceso a la dinámica global. Dichas nociones guardan relación con el nuevo paradigma de desarrollo de los países, basado en la formación del capital humano y en las demandas de los procesos de integración regional que, para esa década, ya se habían consolidado 4 .

Las características del contexto global hacia el año 1990, como, por ejemplo, la creciente demanda intelectual que generaron las nuevas tecnologías y la revalorización de la educación como herramienta generadora de conocimientos vitales para la actividad productiva (Rama, 2006), tuvieron su impacto en la educación superior latinoamericana. Estas características implicaron, por un lado, la masificación de la matrícula universitaria en la región y la ampliación del acceso por la vía privada; por otro lado, el incremento de la oferta de carreras y el surgimiento de algunas de dudosa calidad.

Esto justificó una "reforma educativa", cuyo principal discurso era el del aseguramiento de la "calidad", tanto en educación básica como en educación superior. Con respecto a ese discurso, Rama (2006) destaca el rol que asume el Estado como regulador, buscando la calidad en los programas de enseñanza y en las instituciones a través de mecanismos de evaluación y acreditación.

En tal sentido, identificamos a las políticas de control de la calidad como emergentes del contexto global de los '90, donde fueron cobrando fuerza de ley los procesos de evaluación y

\footnotetext{
${ }^{4}$ La consolidación del proceso de integración regional en el mundo reconoce dos etapas en su evolución, la denominada viejo regionalismo y nuevo regionalismo. El primero, surgió en la década de los 50', en el ámbito bipolar de la Guerra Fría; se consideró cerrado, proteccionista, orientado hacia el interior. El segundo, surgió aproximadamente en los $80^{\prime}$, enmarcado por el proceso de globalización y en un orden mundial multipolar. Para más información sobre este tema, consultar lbáñez, J. (1990).
}

\section{Acreditación Nacional y Regional de las carreras de Ingeniería. EI MARCA como herramienta clave para la integración \\ Silvana Lorena Lagoria \\ DOI : http://dx.doi.org/10.22458/caes.v9i1.1771}


acreditación de la educación superior en los diferentes países de América Latina ${ }^{5}$. Es decir, los sistemas de acreditación de la educación superior surgieron como respuesta a la necesidad de asegurar la calidad de la oferta académica y de las instituciones.

La finalidad principal de la acreditación es controlar, garantizar y dar fe pública ante la sociedad de la calidad de la educación superior. Asimismo, por ser un proceso social y comunitario que requiere la participación de la comunidad educativa en interacción con las autoridades sectoriales que representan a la sociedad, se le atribuye el efecto de contribuir a la construcción de una cultura de la calidad (Mora: 2005; Dias Sobrinho: 2008).

En Argentina, el control de la calidad se consolidó el 20 de julio del año 1995 con la sanción de la Ley de Educación Superior № 24.521 (LES) y la creación de la CONEAU (Comisión Nacional de Evaluación y Acreditación Universitaria) ${ }^{6}$. Mediante la acreditación, el Estado certifica que una determinada carrera o programa de estudio cumple con los estándares mínimos previamente definidos por el Ministerio de Cultura y Educación en consulta con el Consejo de Universidades (LES, Art. 46).

La CONEAU es el órgano oficial encargado de llevar adelante el proceso de acreditación y evaluación de la educación superior y comenzó sus funciones un año después de su creación. Este mismo organismo oficia como ANA (Agencia Nacional de Acreditación) en el proceso de acreditación regional ARCUSUR, donde sus funciones son, entre otras: lanzar la

\footnotetext{
${ }^{5}$ En relación con ello, cabe mencionar la experiencia de Brasil en la evaluación de programas de postgrado que data del año 1951 con la creación de la CAPES (Coordenação de aperfeiçoamento de pessoal de nivel superior) dedicada a dicha tarea, entre otras funciones.

6 CONEAU (Comissão Nacional de Avaliação e Acreditação da Educação Superior) <http://www.coneau.edu.ar)>

Acreditación Nacional y Regional de las carreras de Ingeniería. EI MARCA como herramienta clave para la integración

Silvana Lorena Lagoria

DOI : http://dx.doi.org/10.22458/caes.v9i1.1771
} 
convocatoria en el territorio nacional, aplicar el procedimiento y otorgar la acreditación en función de los estándares regionales.

En el contexto regional, la acreditación se hizo concreta a partir del Mecanismo Experimental de Acreditación de Carreras de Grado (MEXA), que fue definido en junio de 1998 y tuvo vigencia hasta el año 2006, y luego fue reemplazado por el ARCU-SUR (sistema permanente de Acreditación de Carreras Universitarias para el Reconocimiento Regional de la Calidad Académica de las respectivas titulaciones en el Mercosur y los Estados Asociados).

La acreditación en el MEXA (y su continuidad a través del ARCUSUR), se enmarca en el interés del bloque por fortalecer el MERCOSUR Educativo como espacio común de integración. Así, en el Primer Plan Trienal del Sector Educativo del MERCOSUR se planteaba

(...) la compatibilización de los sistemas educativos, el reconocimiento de estudios y homologación de títulos con la finalidad de facilitar la circulación de estudiantes y profesionales de la región y la formulación de propuestas de flexibilización, acreditación y reconocimiento de estudios y títulos (Fernández Lamarra, 2005, p.98).

Gonzales y Castillo (2006) mencionan a la acreditación de carreras en el MERCOSUR como una acción política concreta orientada al fortalecimiento del bloque y a la integración educativa, a partir de la conformación de un espacio de educación común. Así, los propósitos con los que fueron creados los procedimientos de acreditación en el MERCOSUR tuvieron que ver con: contribuir a la conformación de espacio de educación común y a la integración educativa, asegurar la formación de recursos humanos capacitados que contribuyan al desarrollo regional, reconocimiento de títulos de grado a nivel regional, facilitar la movilidad

Acreditación Nacional y Regional de las carreras de Ingeniería. El MARCA como herramienta clave para la integración

Silvana Lorena Lagoria

DOI : http://dx.doi.org/10.22458/caes.v9i1.1771 
académica en la región, estimular los procesos de evaluación con el fin de elevar la calidad educativa.

En relación con estos propósitos de la acreditación tanto en el nivel regional como en el nacional, nuestras investigaciones previas (Barreyro y Lagoria, 2010; Lagoria, 2010; Barreyro, Lagoria, Hizume, 1012; Lagoria 2016) nos permiten establecer algunas reflexiones respecto de los efectos de la acreditación a escala regional y nacional en las carreras de grado que integraron este estudio.

\section{Acreditación, cambio y cultura de la calidad}

En el nivel nacional, nos interesaba saber el modo en que el proceso de acreditación incide en las prácticas institucionales en términos de mejora de la calidad. A la luz de dicho propósito, intentamos detectar cuáles son los resultados visibles y constatables de la acreditación en el orden académico, organizativo y cultural en las carreras seleccionadas para esta investigación.

Recordemos que, como dijimos anteriormente, el proceso de acreditación se propone controlar, garantizar y dar fe pública ante la sociedad de la calidad de la educación superior. Por esto mismo, a este proceso se le atribuye la finalidad de promover y estimular el continuo mejoramiento de la educación superior y se le asocia a la tarea de fomentar la cultura de la calidad.

En el caso de las carreras que integran la muestra, la acreditación nacional se realizó en dos fases. Esto se debe a que, en una primera instancia de evaluación, las carreras no alcanzaron los estándares mínimos de acreditación y, por ello, se les otorgó una acreditación

Acreditación Nacional y Regional de las carreras de Ingeniería. El MARCA como herramienta clave para la integración

Silvana Lorena Lagoria

DOI : http://dx.doi.org/10.22458/caes.v9i1.1771 
válida por tres años con el compromiso de realizar las mejoras necesarias para alcanzar los estándares luego de transcurridos esos tres años ${ }^{7}$.

En la 1ra. fase de acreditación nacional, las carreras analizadas accedieron al Programa para la mejora de las Ingenierías (PROMEI). Este programa significó un elemento fundamental para realizar modificaciones y cumplir con los compromisos asumidos, principalmente en los componentes investigación, biblioteca, equipamiento y docencia.

Sin duda, los planes de mejora del proceso de acreditación nacional fueron un facilitador del cambio en lo que refiere a los componentes mencionados en el párrafo anterior. Esto permitió a las carreras obtener una acreditación por tres años más como resultado de la 2da. fase de acreditación, quedando registrado en las resoluciones correspondientes, donde se expresan los avances y se deja constancia de que las carreras han alcanzado los estándares requeridos.

En el nivel regional, un aspecto directamente relacionado con la acreditación de carreras de grado es la intención del reconocimiento de títulos de grado en el bloque y la movilidad académica en la región. Con relación a ello, en el Manual de Procedimientos del Sistema ARCU-SUR (2008) se aclara que el reconocimiento de la calidad de los títulos de grado otorgados en este Sistema, no confiere de por sí el derecho al ejercicio de la profesión en los demás países. Sin embargo, este reconocimiento será tomado en cuenta como criterio

${ }^{7}$ En Argentina, según la LES n. 24.521, los resultados del proceso de acreditación pueden ser: a) acreditación por 6 años, cuando las carreras cumplen con el perfil previsto; b) acreditación por 3 años, cuando las carreras cumplen con el perfil previsto pero no tiene un ciclo completo de dictado y, por lo tanto, no tienen egresados o no cumplen con el perfil previsto pero tiene estrategias de mejoramiento adecuadas que se transforman en compromisos; y, c) no acreditación, cuando la carrera no cumple con el perfil previsto y sus estrategias de mejoramiento no permiten esperar que se alcancen en un plazo razonable

Acreditación Nacional y Regional de las carreras de Ingeniería. El MARCA como herramienta clave para la integración

Silvana Lorena Lagoria

DOI : http://dx.doi.org/10.22458/caes.v9i1.1771 
común para articular con programas regionales de cooperación, fomento, subsidio, movilidad, entre otras, que beneficien a los sistemas de educación superior en general.

El sentido de la acreditación en el Sistema ARCU-SUR será el de constituirse como criterio común para facilitar el reconocimiento mutuo de títulos o diplomas de grado universitario para el ejercicio profesional en los convenios, tratados o acuerdos bilaterales, multilaterales, regionales o subregionales que se celebren al respecto.

Con relación a estas intenciones de reconocimiento de títulos y movilidad en la región, las entrevistas a informantes claves revelaron que, en general, la ventaja de estar acreditados en el MERCOSUR pasa más por una cuestión de prestigio que por otra cosa, ya que la acreditación no habilita para el ejercicio profesional en otro país del bloque (Lagoria, 2016).

La acreditación regional le brinda un valor agregado (simbólico) al título y a la acreditación nacional. Además, es un requisito para acceder al programa de Movilidad Académica Regional para las Carreras Acreditadas del MERCOSUR (MARCA) ${ }^{8}$ y esto es valorado positivamente por los actores institucionales.

Un aspecto destacable es que, según los entrevistados, el cumplimiento de los procedimientos de acreditación regional no demandaría demasiados esfuerzos mientras se realice de modo coordinado con el proceso nacional. En el caso contrario, el esfuerzo se duplica, como sucedió con la acreditación ARCUSUR de la carrera de Ingeniería Electrónica

${ }^{8}$ El programa de movilidad MARCA lanzó su primera convocatoria en el año 2006 como prueba piloto con la participación de la carrera de Agronomía y, en el año 2007, luego de una evaluación con resultados satisfactorios, fue ampliando hacia otras carreras que contaban con acreditación regional. Más información en: http://programamarca.siu.edu.ar/acerca.php

Acreditación Nacional y Regional de las carreras de Ingeniería. El MARCA como herramienta clave para la integración

Silvana Lorena Lagoria

DOI : http://dx.doi.org/10.22458/caes.v9i1.1771 
de la UNC, donde la acreditación nacional se demoró y fue necesario actualizar el informe realizado anteriormente (Lagoria, 2016).

Hasta el momento de finalización del trabajo de campo de esta investigación (2012 a 2013), no existía un acuerdo para compatibilizar la aplicación de los procedimientos nacionales y regionales de acreditación. Si existiera tal acuerdo y ambas acreditaciones fueran realizadas de manera coordinada es posible que no sólo se disminuyan las exigencias burocráticas del proceso, sino que también se produciría un ahorro en los recursos materiales y humanos que demanda el proceso 9 .

En síntesis, según la experiencia comentada por los actores entrevistados, la acreditación regional en el MERCOSUR no tendría mayor impacto que el de emitir una certificación regional para las carreras, ya que el reconocimiento de títulos de grado en el bloque no es aún una realidad concreta. A ello se suma la posibilidad de las carreras de participar en el programa de movilidad académica MARCA.

A diferencia del MERCOSUR, en el bloque Unión Europea (UE) el proceso de acreditación regional se vive de manera diferente. Las entrevistas realizadas en la UE confirmaron que la acreditación permite al estudiante continuar sus estudios en otro país y ejercer su profesión en cualquier país del bloque de manera certera.

${ }^{9}$ Con relación a este tema, en noviembre de 2015, se aprobó una solicitud presentada por la CONEAU para la implementación simultánea de los procesos de acreditación nacional y regional ARCUSUR (Reunión de la Red de Agencias Nacionales de Acreditación, Acta 02/15).

Acreditación Nacional y Regional de las carreras de Ingeniería. El MARCA como herramienta clave para la integración

Silvana Lorena Lagoria

DOI : http://dx.doi.org/10.22458/caes.v9i1.1771 
Esto encuentra su fundamento en las acciones en educación superior basadas en el criterio de supranacionalidad que adoptó el bloque y que llevó a conformar el Espacio Europeo de Educación Superior (EEES) en la Declaración de Boloña del año 1999.

Entre esas acciones, el aseguramiento de la calidad de la educación superior se materializó en la creación de una serie de organismos regionales vinculados al control de la calidad en el bloque, como es el caso de la European Association for Quality Assurance in Higher Education (ENQA). La ENQA cumple un importante papel en el tema de la acreditación en el EEES junto con EUA (European University Association), EURASHE (European Association of Institutions of Higher Education) y ESIB (European Student Information Bureau o Sindicatos Nacionales de Estudiantes de Europa, en español).

Asimismo, para facilitar la movilidad académica y la equivalencia de títulos, se reestructuró el sistema de educación superior en dos ciclos: grado (Licenciatura) y postgrado (Máster), al que luego se incorporó el doctorado como un tercer ciclo (Reunión de ministros, Berlín 2003) y se creó un sistema común europeo de créditos $(\mathrm{ETCS})^{10}$.

Volviendo la mirada al MERCOSUR, sabemos que, en las condiciones institucionales en que se organiza dicho bloque regional, no es posible pensar el reconocimiento de títulos, el ejercicio profesional y la movilidad de estudiantes y docentes en el modo en que se pone en práctica en la UE. Para que eso ocurra, sería necesario reestructurar y repensar algunas cuestiones del bloque, además de que la naturaleza de un "mercado común" no incluye la supranacionalidad. En ese sentido, las características de la UE como "unión monetaria", y el

10 En la actualidad, Argentina está evaluando la posibilidad de implementar un sistema de créditos con la intención de contribuir a una mayor integración del sistema y de generar más flexibilidad en los planes de estudio. (Origlia, 2016).

Acreditación Nacional y Regional de las carreras de Ingeniería. El MARCA como herramienta clave para la integración

Silvana Lorena Lagoria

DOI : http://dx.doi.org/10.22458/caes.v9i1.1771 
nivel de integración que ello implica, estarían facilitando la movilidad y el reconocimiento de títulos con posibilidades de ejercicio profesional (Lagoria, 2016).

En relación con la construcción de una cultura de la calidad, nuestros entrevistados coincidieron en afirmar que la participación en los procesos de acreditación tanto en el nivel nacional como en el regional despierta el interés propio por brindar una oferta de calidad.

Asimismo, dicha participación contribuye a la toma de conciencia del nivel de calidad de la oferta. Es decir que, el hecho de participar activamente de los procedimientos de acreditación y de visualizar concretamente los resultados, contribuye a que los actores institucionales alcancen a dimensionar aquellos aspectos fuertes de la formación que brindan y las debilidades que deberían atender.

Desde el punto de vista de Harvey y Green (1993) podríamos decir que, en el caso de algunas de las carreras seleccionadas para la muestra, los actores institucionales asumen a la acreditación como "perfección".

La calidad como perfección, se basa en la idea de que las acciones en educación superior se realicen de manera correcta y sin defectos. La mirada está puesta en los procesos institucionales y la idea central es orientar cada etapa de los procesos a través de acciones hacia la perfección, evitando cualquier posible defecto. El concepto de "cultura de la calidad" se vincula a este enfoque en el sentido de que todos los miembros de la organización son responsables de la calidad (Harvey y Green, 1993). Si bien, los actores entrevistados no manifestaron explícitamente esta intención, es posible sugerir que la preocupación genuina

Acreditación Nacional y Regional de las carreras de Ingeniería. El MARCA como herramienta clave para la integración

Silvana Lorena Lagoria

DOI : http://dx.doi.org/10.22458/caes.v9i1.1771 
por mantener un nivel de calidad en la oferta ha estado presente desde tiempos precedentes a la puesta en marcha de los procesos de acreditación.

Por ejemplo, en la carrera de Ingeniería Industrial de la UTN, los entrevistados manifestaron que la calidad fue una preocupación desde los inicios del funcionamiento de la carrera. También comentaron que, desde antes que se implementara la acreditación nacional, sostenían que el control de la calidad es una buena práctica y creían necesario efectuarlo. Este interés los llevó a buscar otros certificados de calidad antes de que se hablara del tema a nivel nacional (certificación del Det Norske Veritas ${ }^{11}$ ).

Esto indica, además, que el interés por la calidad de la oferta no estuvo relacionado sólo con el hecho de responder a la demanda del control estatal. Esta afirmación se funda en las entrevistas, donde los actores institucionales expresaron un interés interno por brindar una oferta de calidad.

Como podemos observar, el cambio de orden cultural en estas carreras es tanto una construcción del contexto de las decisiones macro políticas, como un resultado de los intereses que circulan entre los miembros de la institución. De este modo, es posible visualizar el control de la calidad como una construcción simbólica propia de los individuos que las producen y transmiten en contextos estructurados e históricamente específicos (Thompson, 1990).

11 Det Norske Veritas (DNV) fue fundada en 1864 como una Sociedad de Clasificación de ámbito mundial con sede en Noruega. Se trata de una fundación independiente cuyos objetivos son salvaguardar la vida y propiedades del medio marino y es, a la vez, un proveedor líder en servicios de gestión de riesgos. Para más información dirigirse al sitio: https://www.dnvgl.com

Acreditación Nacional y Regional de las carreras de Ingeniería. El MARCA como herramienta clave para la integración

Silvana Lorena Lagoria

DOI : http://dx.doi.org/10.22458/caes.v9i1.1771 
Esto sugiere que la calidad de la oferta es un tema de preocupación instalado en los actores que atraviesan los procesos de acreditación, al menos en quienes participaron de las entrevistas, principalmente, los integrantes de los equipos de gestión y algunos profesores.

La construcción de una cultura de la calidad es un proceso social en el que intervienen no sólo los actores de las instituciones universitarias, sino todos aquellos involucrados en la acreditación, ya sea que pertenezcan al ámbito nacional o regional de acreditación. De este modo, la cultura de la calidad se va configurando no desde cada actor individual, sino desde la "acción colectiva" (Crozier y Friedberg, 1990) en la que todos participan.

\section{Continuidades y rupturas entre la acreditación regional y nacional}

Una cuestión importante que los actores mencionaron en las entrevistas es el hecho de que los procesos de acreditación regional y nacional se implementaron de manera paralela en el tiempo. Esto, de cierto modo, facilitó la tarea de responder a uno y otro proceso.

Sin embargo, al relacionar el proceso de acreditación regional con el nacional, los actores detectaron ciertas "incoherencias de la acreditación", tal como lo mencionó uno de los entrevistados.

Al respecto, se constató en documentos y entrevistas que una de las carreras de Ingeniería Electrónica de la muestra, fue acreditada por cinco años en MEXA y no fue acreditada en el nivel nacional, siendo que ambos procesos se desarrollaron de manera paralela en el tiempo. Este hecho llamó la atención de los actores institucionales, ya que el organismo encargado de llevar adelante el proceso y de otorgar la acreditación en ambos niveles (regional y

Acreditación Nacional y Regional de las carreras de Ingeniería. El MARCA como herramienta clave para la integración

Silvana Lorena Lagoria

DOI : http://dx.doi.org/10.22458/caes.v9i1.1771 
nacional) es el mismo, la CONEAU, aunque el comité de Pares haya estado conformado de manera diferente en cada caso.

Asimismo, al indagar las resoluciones que expresan los resultados obtenidos por las carreras acreditadas en el nivel regional, los entrevistados destacaron como hecho confuso que las acreditaciones en MEXA y ARCUSUR se otorgaran de manera directa por 5 y 6 años respectivamente, mientras que la acreditación nacional les demandaba exigencias mayores. Esto nos remite a un análisis de los estándares evaluativos que no alcanzamos a indagar en la investigación originaria de la que parte este artículo.

Del análisis documental, también se constató que, en la acreditación regional, no se asumieron compromisos de mejora por parte de la carrera como en la acreditación nacional. Sin embargo, los pares evaluadores realizaron recomendaciones sobre las dimensiones que se consideraron débiles y sobre las que se podían mejorar.

Las incongruencias que mencionan los actores institucionales respecto al proceso de acreditación nacional considerado a la par de la acreditación regional, nos hace pensar que las razones de las mismas son diversas. Dichas razones podrían incluir desde una mayor flexibilidad de criterios en la acreditación regional, hasta una cuestión pragmática de economizar recursos. Para un análisis en profundidad de este tema sería interesante realizar un estudio comparado de los estándares de acreditación del bloque regional y del organismo estatal para alcance nacional.

Frente a esta realidad que plantean los actores y en base a los hallazgos de esta investigación, nos surgen algunas inquietudes que abren al debate. Por ejemplo: ¿se justifica

Acreditación Nacional y Regional de las carreras de Ingeniería. El MARCA como herramienta clave para la integración

Silvana Lorena Lagoria

DOI : http://dx.doi.org/10.22458/caes.v9i1.1771 
el gasto y movilización de actores que demanda un proceso de acreditación para otorgarle un valor simbólico a la acreditación nacional, que por sí misma es rigurosa y exigente? ¿cuál es el impacto de los beneficios de la acreditación regional para el egresado? ¿hasta qué punto es necesaria una acreditación regional con las características del ARCUSUR para acceder a programas de intercambio, cuando la movilidad de alumnos y profesores es una realidad muy instalada en nuestra sociedad actual y a la que se puede acceder por diferentes vías?

Respecto a este último interrogante, es necesario tener en cuenta que el programa MARCA, se financia exclusivamente con el presupuesto universitario. La Convocatoria 2015-2016 estipula que el financiamiento del Programa será:

Para los estudiantes: La Universidad de Origen cubrirá los costos del pasaje y seguro. La Universidad de Destino cubrirá los costos de alojamiento y manutención. Para los docentes y coordinadores: La Universidad de Origen cubrirá los costos de alojamiento y manutención, pasaje y seguro (aunque no está acordado entre los países, dependerá de decisiones posteriores) (MERCOSUR EDUCATIVO, 2015: p.8).

Desde allí, se entiende que el bloque MERCOSUR, no destina fondos ni planes de financiamiento para llevar adelante la movilidad académica. Lo mismo ocurre con otras acciones de internacionalización en el bloque, como, por ejemplo, con la experiencia de la Agrupación de Universidades del Grupo Montevideo (AUGM) ${ }^{12}$ que es una agrupación auto gestionada de universidades públicas de Argentina, Bolivia, Brasil, Chile, Paraguay y Uruguay que funcionan a modo de Red.

${ }^{12}$ AUGM (Asociación de Universidades Grupo Montevideo) http://grupomontevideo.org/sitio/

Acreditación Nacional y Regional de las carreras de Ingeniería. EI MARCA como herramienta clave para la integración

Silvana Lorena Lagoria

DOI : http://dx.doi.org/10.22458/caes.v9i1.1771 
Esta agrupación nació en agosto de 1991 por iniciativa de las universidades de hacer frente a los desafíos comunes de la región. Desde entonces desarrollan actividades de cooperación e internacionalización de manera autónoma y desvinculada administrativamente del bloque económico MERCOSUR, entre ellas, la movilidad estudiantil a través del programa "ESCALA Estudiantes de Grado" y "ESCALA Estudiantes de Postgrado" destinado a la promoción de la movilidad de los estudiantes regulares de maestrías y doctorados.

Llegado a este punto que se abre al debate, parece necesario reflexionar acerca del sentido de la acreditación regional y revisar sus propósitos para reformularlos en relación con lo que se vislumbra como posible.

Viendo el panorama que se plantea, tal vez una opción para optimizar el sentido de la acreditación sería pensar en la autogestión del ARCUSUR para su funcionamiento independiente del MERCOSUR, como una iniciativa propia de las universidades y de las agencias acreditadoras funcionando a modo de "red". De este modo, se podrían plantear objetivos específicos para el mecanismo regional de control de la calidad, que orienten el proceso de acreditación para lograr fines pensados por y para la educación, sin la pretensión ni la exigencia de ser parte de un bloque cuyo interés primordial estuvo orientado siempre hacia el crecimiento económico de la región.

\section{Conclusiones}

Nuestra investigación, sin llegar a ser un estudio de impacto, pretendió indagar y analizar comparativamente lo que sucede en las instituciones y en las carreras como consecuencia de la implementación de los sistemas de acreditación de carreras universitarias en el marco de la Ley de Educación Superior № 24.521 y de las disposiciones del MERCOSUR en

Acreditación Nacional y Regional de las carreras de Ingeniería. El MARCA como herramienta clave para la integración

Silvana Lorena Lagoria

DOI : http://dx.doi.org/10.22458/caes.v9i1.1771 
materia educativa (procesos MEXA y/o ARCUSUR). El foco del análisis estuvo puesto en lo que sucede en el nivel operativo de las prácticas y las modificaciones que se registran en respuesta al proceso de acreditación nacional y regional.

Como reflexión final, podemos afirmar sin dudas que la acreditación en el ámbito nacional contribuye al control de la calidad, a la mejora académica y a la promoción de una cultura de la calidad. No obstante, debemos reconocer que los cambios y mejoras verificados en las carreras no son un resultado generado exclusivamente a partir de dichos procesos.

Las mejoras realizadas a partir del proceso de acreditación nacional son verificables tanto en los documentos como en las entrevistas realizadas a actores clave. Sin embargo, la repercusión de los procesos de acreditación no es independiente de otros factores propios del contexto institucional, tal como se manifiesta en el análisis realizado.

Recordemos que, en algunas de las carreras seleccionadas, los documentos y las entrevistas a actores ponen de manifiesto un interés propio y genuino por controlar la calidad de la oferta a través de experiencias auto-gestionadas que fueron previas e independientes a la implementación de los procesos de acreditación en nuestro país.

En el nivel de regional, podemos decir que la acreditación le otorga un mayor prestigio a la carrera acreditada en MEXA y/o ARCUSUR. Sin embargo, no se constataron mejoras vinculadas a la acreditación regional.

Es posible que esto se deba a la dinámica del procedimiento y a que, en el marco de las políticas a nivel macro, no se destinan fondos para planes de mejoras en educación superior.

Acreditación Nacional y Regional de las carreras de Ingeniería. El MARCA como herramienta clave para la integración

Silvana Lorena Lagoria

DOI : http://dx.doi.org/10.22458/caes.v9i1.1771 
En este punto fue muy relevante el aporte de los actores clave, quienes, en las entrevistas, establecieron claras diferencias entre los beneficios obtenidos de la acreditación nacional y regional.

Respecto de la cultura de la calidad podríamos decir que, en tanto ésta se constituye de pautas socialmente construidas que se sostienen en el tiempo y que se hacen carne en los actores institucionales (Crozier y Friedberg, 1990), el hecho de que las carreras hayan atravesado por varias fases de acreditación y en diferentes niveles (nacional y regional), ha contribuido al fortalecimiento de una "cultura de la calidad".

En ese sentido, la cultura de la calidad se va consolidando progresivamente en la medida en que las carreras van atravesando las diferentes fases e instancias de acreditación. Es posible afirmar también que, la acreditación regional aporta a ello. Así se pone en evidencia la influencia del factor "tiempo" en la construcción de patrones estructurales a los que refiere Krostch (2003).

En suma, tanto los procesos de acreditación en el nivel nacional como en el regional contribuyen a estimular la construcción de la cultura de la calidad, ya que involucran a todos los actores que se comprometen con la tarea, siempre que haya continuidad y no queden como un hecho aislado.

En cuanto al "cambio" vinculado a la idea de "mejora", se verificó sólo en el proceso de acreditación nacional vinculado a un factor puntual: los planes de mejora (PROMEI) implementados por el gobierno nacional.

Acreditación Nacional y Regional de las carreras de Ingeniería. El MARCA como herramienta clave para la integración

Silvana Lorena Lagoria

DOI : http://dx.doi.org/10.22458/caes.v9i1.1771 
Otro aspecto clave que surge del análisis realizado se refiere a que el programa MARCA se rige como una herramienta clave vinculada a los objetivos del bloque regional para el sector educación, principalmente, a los referidos a la cooperación interinstitucional, a la movilidad académica y a la integración educativa. El sostenimiento del MARCA a través del tiempo y la participación de las carreras acreditadas son datos importantes que dan cuenta de ello.

Asimismo, los avances respecto al MARCA avizoran un futuro prometedor. Respecto al financiamiento por parte del MERCOSUR relacionado a la acreditación regional, actualmente se está tratando la posibilidad de que el Sistema Integrado de Movilidad (SIMERCOSUR) se integre a las estructuras de financiamiento y gestión del programa MARCA. Según la Lic. Mercedes Gandola, se crearon tres puestos de la Unidad Técnica de Educación y se están realizando las convocatorias para cubrir los cargos.

De este modo, el bloque regional financiaría políticas vinculadas a la acreditación regional. Siguiendo una misma línea de investigación, será interesante analizar en un futuro las posibilidades que se abren a partir de estos nuevos financiamientos y los efectos que dichas políticas ejercen sobre el interés de los actores institucionales.

En cuanto a nuestra mirada del proceso de acreditación en la UE y MERCOSUR desde las prácticas, hemos visto que en uno y otro bloque significan realidades absolutamente diferentes. El sentido del proceso a nivel regional en la UE tiene alcances, objetivos y desarrollos diferentes, así como las reinterpretaciones de quienes los llevan adelante en las instituciones.

Acreditación Nacional y Regional de las carreras de Ingeniería. El MARCA como herramienta clave para la integración

Silvana Lorena Lagoria

DOI : http://dx.doi.org/10.22458/caes.v9i1.1771 
Siguiendo la perspectiva de Krotsch (2003), tuvimos presente que tanto la implementación de políticas en educación como su análisis debe tomar en cuenta lo local. De modo contrario, existe la posibilidad de que no se produzcan los efectos esperados desde su elaboración hasta su puesta en marcha en el nivel de las prácticas en el caso de su implementación; o, que se pretenda pensar que una política que resultó exitosa en un lugar pueda ser aplicada directamente en otro con resultados similares, en el caso del análisis con miras a ser aplicada en otra realidad.

Por tal motivo, consideramos junto con Tello (2015) que, el proceso de integración de la Educación Superior o de creación de un espacio de Educación Superior en nuestra región, debe pensarse como un proceso de intercambio desde la solidaridad académica. En ese sentido, y considerando el análisis precedente, tal vez sea necesario realizar un ajuste de las expectativas que se esperan alcanzar con el proceso ARCUSUR desde una mirada de lo posible y reforzar un perfil educativo por sobre el económico.

Por último, deseamos destacar que el marco conceptual construido para esta investigación resultó pertinente para el análisis de los aspectos culturales y del cambio. Asimismo, dicho marco permitió analizar las prácticas institucionales, sus transformaciones y ajustes en estrecha vinculación con los efectos que vienen ejerciendo en el sistema educativo las macropolíticas estatales y regionales. El hecho de poder investigar y analizar estos aspectos desde las prácticas de los actores institucionales permite interpelar a dichas políticas desde los sentidos que le atribuyen los sujetos que le dan vida. Es decir, permiten otro modo de hacer política, desde la toma de conciencia de sus potencialidades y efectos, para participar en la arena de negociaciones y conducirlas en el accionar cotidiano de la escuela con criterio y responsabilidad social.

Acreditación Nacional y Regional de las carreras de Ingeniería. El MARCA como herramienta clave para la integración

Silvana Lorena Lagoria

DOI : http://dx.doi.org/10.22458/caes.v9i1.1771 


\section{Referencias}

ARCUSUR (2015). Reunión de la Red de Agencias Nacionales de Acreditación. Acta 02/15.

Argentina (1995). Ley de Educación Superior n. 24.521

Ball, S. (2002). Textos, discursos y trayectorias de la política: la teoría estratégica. Revista Páginas, 2 (2 y 3), 19-33. (traducción Estela M. Miranda)

Barreyro, G. y Lagoria, S. (2010). Acreditação da educação superior na América Latina: os casos da Argentina e do Brasil no contexto do Mercosul. Revista PROLAM 1, 7-27. Recuperado el 8 -6- 2014 de: http://www.usp.br/prolam/cadernos.htm

Barreyro, G., Lagoria, S. y Hizume G. (2012). As Agências Nacionais de Acreditação no Sistema ARCU-SUL: primeiras considerações. Revista da Avaliação da Educação Superior. 20(1). Campinas; Sorocaba, SP. pp. 49-72. Recuperado el 28-9-2016 de http://periodicos.uniso.br/ojs/index.php?journal=avaliacao\&page=article\&op=view\&path []=2165

Crozier, M. y Friedberg, E. (1990). El actor y el sistema, las restricciones de la acción colectiva. México. Editorial Alianza Mexicana.

Dias, S. (2008). Acreditação da educação superior (mimeografado).

Lamarra, N. y Mora, G. (org.) (2005). Educación Superior: convergencia entre América Latina y Europa. Procesos de evaluación y acreditación de la calidad. 1르. Ed. Buenos Aires: Ed. Eduntref.

Gandola, M. (2016). (Ministerio de Educación y Deportes) Entrevista concedida a Silvana Lagoria.

Geertz, C. (1992). La interpretación de las culturas. Barcelona: Gedisa.

González, L. E. y Castillo, D. (2006). La educación en el siglo XXI: Estudios, investigaciones, estadísticas y evaluaciones educacionales. Estudio Analítico Comparativo del Sistema Educacional de MERCOSUR-Educación Superior. Proyecto BRA/04/049 - Informe Final INEP-PNUD. Chile.

Acreditación Nacional y Regional de las carreras de Ingeniería. El MARCA como herramienta clave para la integración

Silvana Lorena Lagoria

DOI : http://dx.doi.org/10.22458/caes.v9i1.1771 
Green, A. (2003). Education, Globalisation and the role of Comparative Research. London Review of Education, 1(2). pp. 83-98. Recuperado el 10-05-2015 de: http://eprints.ioe.ac.uk/5488/

Harvey, L. y Green, D. (1993). Defining quality. Assessment and Evaluation in Higher Education, 18(1), 9-34.

Ibáñez, J. (1990). El nuevo regionalismo latinoamericano en los años noventa. Recuperado el 28-4-2010 de: www.reei.org/reei.1/lbanezag.reei. PDF

Krotsch, P. (2003). Educación superior y reformas comparadas. Buenos Aires: Universidad Nacional de Quilmes.

Lagoria, S. L. (2010). Acreditación de la Educación Superior en el MERCOSURposibilidades de integración. (Tesina de conclusión del Curso Latino-Americano de Especialização em Políticas Públicas e Avaliação do Ensino Superior. Universidade Federal do Paraná/Universidade Federal da Integração Latinoamericana (UNILA)), Foz do Iguaçu. p. 69.

Lagoria, S. L. (2016). Acreditación de carreras de grado en el ámbito nacional y regional. El caso de las ingenierías. (Tesis Doctoral (Doctorado en Estudios Sociales de América Latina) Centro de Estudios Avanzados de la Universidad Nacional de Córdoba), Córdoba. p. 312.

Mainardes, J. y Marcondes, M. I. (2009). Entrevista con Stephen J. Ball: um diálogo sobre justiça social, pesquisa e política educacional. Educação \& Sociedade, 30 (106), pp.303-318.

MERCOSUR Educativo (2015). MARCA. Termos de referência da chamada 2015-2016. Setor educativo do Mercosul. Comissão Regional de Coordenação do Ensino Superior. 2015. Recuperado el 14-09-2016 de: http://programamarca.siu.edu.ar/documentos/2015-2016/TDR_MARCA_2015-2016.pdf

Miranda, E. (2011). Una "caja de herramientas" para el análisis de la trayectoria de la política educativa. La perspectiva de los ciclos de la política (Policy Cycle Approach). En: Miranda, E. y Bryan, N. (Org.) (Re) Pensar a Educação Pública: contribuições da Argentina e do Brasil. Córdoba. Ed. FFyH-UNC. pp. 105-128.

Acreditación Nacional y Regional de las carreras de Ingeniería. EI MARCA como herramienta clave para la integración

Silvana Lorena Lagoria

DOI : http://dx.doi.org/10.22458/caes.v9i1.1771 
Mora, J. G. (2005). La evaluación y acreditación en la Unión Europea, en J.G. Mora y N. Fernández Lamarra, Educación Superior. Convergencia entre América Latina y Europa, Eduntref, Buenos Aires. pp. 21-30.

Origlia, G. (2016, 07 de Septiembre). Impulsan un acuerdo entre universidades para validar materias. La Nación. Recuperado el 30-12-2016 de: http://www.lanacion.com.ar/1935288-impulsan-un-acuerdo-entre-universidades-paravalidar-materias

Rama, C. (2006). La tercera reforma de la educación superior en América Latina. Buenos Aires: FCE.

Stenhouse, L. (1990). Conducción, análisis y presentación del estudio de casos en la investigación educativa y evaluación. En Martínez, J. (1990). Hacia un enfoque interpretativo de la enseñanza, 69 - 84. Granada: Universidad de Granada.

Tello; C. (2015). Políticas educativas, educación superior y proceso de Bolonia en Latinoamérica. Crítica Educativa. Sorocaba/SP, 1(1), 80-97. Recuperado el 30-102016 de: http://oaji.net/articles/2016/2991-1455533181.pdf

Thompson, J. B. (1990). Ideology and Modern Culture. Critical Social Theory in the Era of Mass Communication. Stanford, California/ Stanford: University Press.

Yang, R. (2010). La comparación de políticas. En: M. Bray, B. Adamson \& M. Mason: Educación comparada: Enfoques y Métodos. Buenos Aires. Granica. pp. 295-320.

Acreditación Nacional y Regional de las carreras de Ingeniería. EI MARCA como herramienta clave para la integración

Silvana Lorena Lagoria

DOI : http://dx.doi.org/10.22458/caes.v9i1.1771 